\title{
Time-Domain Fluorescence Lifetime Imaging Microscopy: A Quantitative Method to Follow Transient Protein-Protein Interactions in Living Cells
}

\author{
Sergi Padilla-Parra, Nicolas Audugé, Marc Tramier, and Maïté Coppey-Moisan
}

\begin{abstract}
Quantitative analysis in Förster resonance energy transfer (FRET) imaging studies of protein-protein interactions within live cells is still a challenging issue. Many cellular biology applications aim at the determination of the space and time variations of the relative amount of interacting fluorescently tagged proteins occurring in cells. This relevant quantitative parameter can be, at least partially, obtained at a pixel-level resolution by using fluorescence lifetime imaging microscopy (FLIM). Indeed, fluorescence decay analysis of a two-component system (FRET and no FRET donor species), leads to the intrinsic FRET efficiency value $(E)$ and the fraction of the donor-tagged protein that undergoes FRET $\left(f_{\mathrm{D}}\right)$. To simultaneously obtain $f_{\mathrm{D}}$ and $E$ values from a two-exponential fit, data must be acquired with a high number of photons, so that the statistics are robust enough to reduce fitting ambiguities. This is a time-consuming procedure. However, when fast-FLIM acquisitions are used to monitor dynamic changes in protein-protein interactions at high spatial and temporal resolutions in living cells, photon statistics and time resolution are limited. In this case, fitting procedures are unreliable, even for single lifetime donors. We introduce the concept of a minimal fraction of donor molecules involved in FRET $\left(m f_{\mathrm{D}}\right)$, obtained from the mathematical minimization of $f_{\mathrm{D}}$. Here, we discuss different FLIM techniques and the compromises that must be made between precision and time invested in acquiring FLIM measurements. We show that $m f_{\mathrm{D}}$ constitutes an interesting quantitative parameter for fast FLIM because it gives quantitative information about transient interactions in live cells.
\end{abstract}

During the last 40 years, Förster resonance energy transfer (FRET) has been used to understand a great variety of molecular interactions, both in vitro and in vivo. Advances in different photonic imaging techniques and the development of fluorescent probes, and particularly fluorescent proteins (FPs) (Tsien 1998; Shaner et al. 2005), have made FRET microscopy an extremely useful methodology. Protein-protein interactions in living cells can be directly monitored using FRET. This aspect is critical to improve our understanding of different processes occurring in vivo (biochemical protein cascades) and, if it is performed quantitatively, to build or to improve biological mathematical models (Tuszynski et al. 2006). A quantitative parameter of FRET is the quantum yield of the energy transfer process $(E)$. Donor fluorescence lifetime decreases because of energy transfer in the excited state, and the percentage of the decrease is equal to $E$. The determination of FRET efficiency by fluorescence lifetime measurements is advantageous in living cell studies because the fluorescence lifetime is independent of the fluorophore concentration and the excitation light path—parameters that are

Adapted from Imaging: A Laboratory Manual (ed. Yuste). CSHL Press, Cold Spring Harbor, NY, USA, 2011.

(C) 2015 Cold Spring Harbor Laboratory Press

Cite this introduction as Cold Spring Harb Protoc; doi:10.1101/pdb.top086249 
unknown in cells under the microscope. Other quantitative FRET techniques based on steady-state intensity allow determination of the apparent FRET efficiency, $E_{\text {app }}$ (Gordon et al. 1998; Hoppe et al. 2002; Berney and Danuser 2003; Elangovan et al. 2003; Stockholm et al. 2004; van Rheenen et al. 2004; Zal and Gascoigne 2004; Wlodarczyk et al. 2008). $E_{\text {app }}$ depends directly on the product of two parameters: the intrinsic FRET efficiency value $(E)$ and the fraction of the donor that undergoes FRET $\left(f_{\mathrm{D}}\right)$ (Hoppe et al. 2002). Steady-state intensity-based approaches are not able to obtain $f_{\mathrm{D}}$ out of $E_{\text {app }}$ (Neher and Neher 2004). To determine $f_{\mathrm{D}}$, the intrinsic FRET efficiency $(E)$ must be calculated independently. Fluorescence lifetime imaging microscopy (FLIM) is a well-established technique to determine the fluorescence kinetics of the donor emission for FRET measurements (Verveer et al. 2000; Emiliani et al. 2003; Peter et al. 2005). In FLIM, using the mean lifetime does not require a high number of measured photons. However, to simultaneously obtain $f_{\mathrm{D}}$ and $E$ values from the fit, fluorescence decay analysis must be performed with two or more components, and data have to be acquired with the highest number of photon counts so that statistics are robust enough to reduce fitting ambiguities. Using time-correlated single-photon counting (TCSPC), a high temporal resolution of the fluorescence kinetics (few tens of picoseconds) can be achieved. Several minutes of data acquisition are necessary, however, to obtain sufficient photon statistics per pixel. Such long acquisition times are incompatible with high spatiotemporal resolution of quantitative FRET images. A fast-gated charge-coupled device (CCD) camera combined with a pulsed, wide-field, or pseudowidefield excitation (TriM-FLIM system) can be used to acquire fluorescence decays faster than with the TCSPC method but at the expense of a smaller temporal resolution. To acquire images as fast as possible, a small number of time-gated images together with a low value of photon counts are required. Note, however, that under these conditions, the quality of a double-exponential fit is far from optimal. However, if we consider a two-component system with a narrow distribution of $E$, the concept of a minimal percentage of donor molecule involved in FRET $\left(m f_{\mathrm{D}}\right)$ can be calculated without fitting directly from the mean fluorescence lifetime of the donor (in the absence and in the presence of the acceptor). $m f_{\mathrm{D}}$ is an interesting approach because it provides information about a known threshold of interacting donor protein and is related to the relative concentration of the interacting protein.

\section{PRINCIPLES OF FRET QUANTIFICATION BY TIME-DOMAIN FLIM}

\section{Theory of FRET}

FRET (or, more correctly, Förster-type resonance energy transfer; Förster 1948) is a nonradiative process that occurs between the excited state of a donor and the ground state of an acceptor.

\section{The Transfer Rate}

The rate of energy transfer $\left(k_{\mathrm{t}}[r]\right)$ from the donor to the acceptor is defined by the following equations (Valeur 2002; Lakowicz 2006):

$$
k_{\mathrm{t}}(r)=1 / \tau_{\mathrm{D}}\left(R_{0}^{6} / r^{6}\right),
$$

where $R_{0}$ is the Förster radius, $r$ is the distance between donor and acceptor, and $\tau_{\mathrm{D}}$ is fluorescence lifetime of the donor in the absence of the acceptor. Because the Förster radius is defined as the distance at which FRET is $50 \%$ efficient, if $R_{0}=r$, the rate transfer will be the same as the decay rate of the donor. $R_{0}$ can be defined as

$$
R_{0}=0.211 \cdot\left[\kappa^{2} n^{-4} Q_{D} J\right]^{1 / 6}(\AA),
$$

with

$$
J=\int \varepsilon_{\mathrm{A}}(\lambda) \cdot f_{\mathrm{D}}(\lambda) \cdot \lambda^{4} \cdot d \lambda / \int f_{\mathrm{D}}(\lambda) \cdot d \lambda,
$$


S. Padilla-Parra et al.

and $\kappa^{2}$ is derived from

$$
\kappa^{2}=\left(\cos \theta-3 \cos \theta_{\mathrm{D}} \cos \theta_{\mathrm{A}}\right)^{2},
$$

where $n$ is the refractive index, $Q_{\mathrm{D}}$ is the fluorescence quantum yield of the donor, $\kappa$ is the parameter related to the orientation of donor and acceptor (Dale et al. 1979; Cheung 1991), $\varepsilon_{\mathrm{A}}(\lambda)$ is the acceptor absorption spectrum, and $f_{\mathrm{D}}(\lambda)$ is the donor emission spectrum. Note that $\theta$ is the angle between the emission transition dipole of the donor and the absorption transition dipole of the acceptor, and $\theta_{\mathrm{D}}$ and $\theta_{\mathrm{A}}$ are the angles between the two dipoles and the vector that goes from the donor to the acceptor (Lakowicz 2006). Förster distances are reported in literature for a value of $\kappa^{2}=2 / 3$.

\section{The Efficiency of Energy Transfer}

We can define the efficiency by calculating the proportion of photons absorbed in the donor versus the excitation transferred to the acceptor:

$$
E=k_{\mathrm{T}} /\left(k_{\mathrm{T}}+k_{\mathrm{D}}\right),
$$

where $k_{\mathrm{D}}$ is the sum of all of the relaxation pathways (Fig. 1) of the excited donor other than FRET.

Experimentally, the transfer efficiency is calculated by using the lifetime of the donor alone or in the presence of the acceptor, applying a lifetime methodology:

$$
E=1-\frac{\tau_{\mathrm{F}}}{\tau_{\mathrm{D}}}
$$

in which $\tau_{\mathrm{F}}$ and $\tau_{\mathrm{D}}$ are the lifetimes of the donor in the presence of the acceptor and the donor alone, respectively. Observe that $\tau_{\mathrm{F}}$ and $\tau_{\mathrm{D}}$ are defined on the basis of the following rate constants:

$$
\begin{aligned}
\tau_{\mathrm{F}} & =\frac{1}{k_{\mathrm{r}}+k_{\mathrm{nr}}+k_{\mathrm{T}}}, \\
\tau_{\mathrm{D}} & =\frac{1}{k_{\mathrm{r}}+k_{\mathrm{nr}}} .
\end{aligned}
$$

\section{Fluorescence Decay of the Donor}

Fluorescence is a radiative process (rate constant $k_{\mathrm{r}}$ ), which takes place when molecules excited by light absorption revert to their original state by light emission. Fluorescence is a very brief and transient phenomenon (typically occurring in the picosecond to nanosecond range), in which the molecules in the excited state $S_{1}$ release energy radiatively as they return to their unexcited state $S_{0}$ (Fig. 1). The kinetics of the fluorescence decay (rate constant $k=k_{\mathrm{nr}}+k_{\mathrm{r}}$ ) depends on the relative proportion of the various pathways for returning to the ground state. In time-domain $(\mathrm{td})$ methods, a pulsed excitation source is used to excite the sample. The aim is to acquire the fluorescence decay after the laser pulse. For a single fluorophore in a homogeneous environment, the fluorescence decay can be written as

$$
i(t)=\left(k_{\mathrm{r}}\right)\left[M^{*}\right]=k_{\mathrm{r}}\left[M^{*}\right]_{0} \exp (-t / \tau),
$$

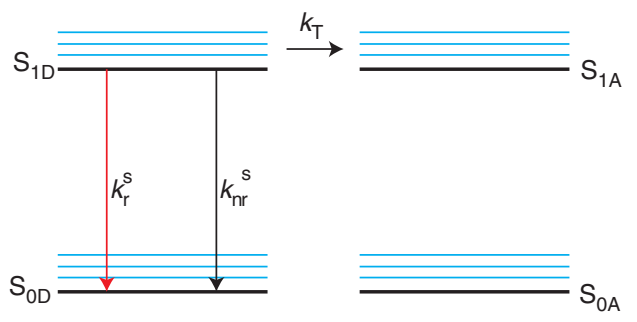

FIGURE 1. Modified Perrin-Jablonski diagrams showing photonic processes occurring in the donor and the FRET process. This diagram takes into consideration the process of FRET in which the donor transfer is indicated with a horizontal black arrow $\left(k_{\mathrm{T}}\right)$. One can see the competition between two pathways of relaxation $\left(k_{\mathrm{r}}\right.$, the radiative process, and $k_{\mathrm{nr}}$, the nonradiative energy dissipation). 
where $\left[M^{*}\right]$ is the concentration of molecules in the excited state and $\tau$ is the lifetime of the excited state. Equation 9 defines the fluorescence decay profile of the donor in the absence of the acceptor.

Now, if we consider a donor-acceptor interaction in which not all of the donor is engaged in the process of FRET, then

$$
i(t)=\left(k_{\mathrm{r}}\right)\left[M^{*}\right]=k_{\mathrm{r}}\left([D]_{0} \exp \left(-t / \tau_{\mathrm{D}}\right)+[D A]_{0} \exp \left(-t / \tau_{\mathrm{F}}\right)\right)
$$

applies. In the above expression, the process of energy transfer is taken into account, and a discrete double exponential describes the fluorescence decay of the donor in the presence of the acceptor. Equation 10 assumes that there is only one orientation that enables the energy transfer to occur. If we normalize the amplitudes of both preexponential factors to 1 , we can introduce the concept of the fraction of the interacting donor $\left(f_{\mathrm{D}}\right)$, and the last equation simplifies to

$$
i(t)=\left(1-f_{\mathrm{D}}\right) \exp \left(-t / \tau_{\mathrm{D}}\right)+\left(f_{\mathrm{D}}\right) \exp \left(-t / \tau_{\mathrm{F}}\right),
$$

where $f_{\mathrm{D}}$ is a parameter that is particularly interesting in the study of the interactions of a related protein in cellular biology. td-FLIM allows for the simultaneous calculation of the fluorescence lifetime of the donor and the other important parameters that describe the fluorescence decay (preexponential factors and, hence, $f_{\mathrm{D}}$ ) pixel by pixel. This makes this technique extremely useful because it retrieves information about the location and the extent of the interaction under study.

\section{Time-Domain Picosecond FLIM: TCSPC-FLIM}

This method is based on TCSPC detection. For each acquired photon, the time between the excitation pulse and the detection of the photon is measured and is used to sample the detected photons (Fig. 2).

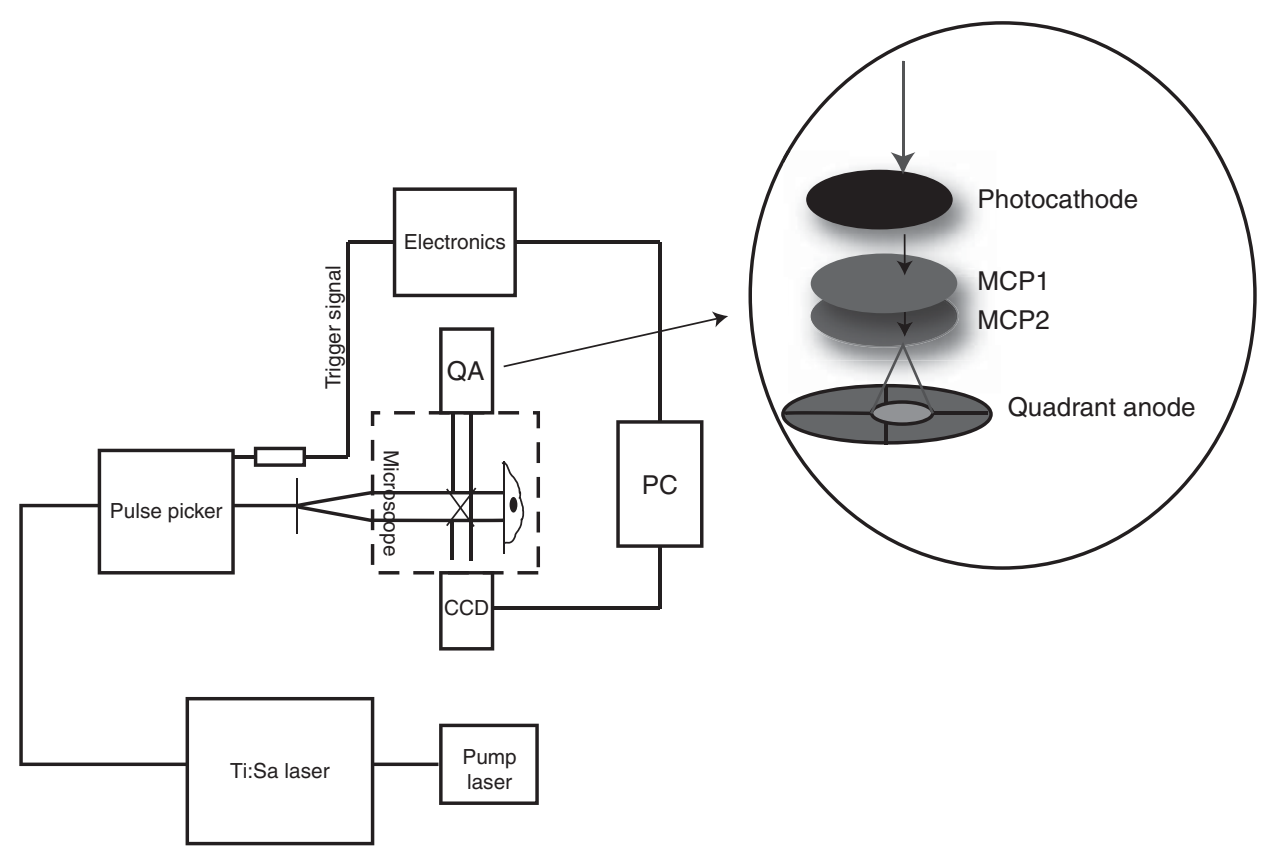

FIGURE 2. Time-domain picosecond TCSPC-FLIM setup. The inset on the right shows the components of the quadrantanode detector. QA, Quadrant anode; PC, personal computer; CCD, charge-coupled device; MCP1, microchannel plate1; MCP2, microchannel plate 2 . 
Excitation: Mode-locked titanium:sapphire lasers can deliver picosecond (1 psec) or femtosecond (100 fsec) pulses and can be tuned between 760 and $980 \mathrm{~nm}$. They can be used for two-photon excitation or for one-photon excitation after frequency doubling. Pulsed laser diodes (1 psec) are now also available for one-photon excitation at 440, 470, and $635 \mathrm{~nm}$ and longer wavelengths. Another option for pulsed excitation is supercontinuum sources.

Microscope and detection: Conventional confocal microscopes have an external port to couple an infrared or visible-light pulsed laser. The laser beam is scanned through the sample, and the single photons are detected by a photomultiplier tube with a time resolution corresponding to 10 psec. Avalanche photodiodes are also used because they are more sensitive in the red part of the spectrum; however, they provide a lower time resolution for the corresponding fluorescence decay. A computer card (e.g., Becker \& Hickl GmbH or Picoquant GmbH) integrates delay time measurements (between emitted photons and excitation pulses) and scan position. Wide-field microscopy can also be used (an approach usually used in our laboratory). In this case, the laser beam of a titanium:sapphire laser (after passing through a frequency doubler and a pulse picker, which provide a reduced repetition rate of $4 \mathrm{MHz}$ ) is coupled to an inverted microscope. A quadrant-anode detector (Europhoton $\mathrm{GmbH}$ ) provides information relative to the temporal and spatial correlation times of each single photon counted. Fluorescence decays are acquired by counting and sampling single emitted photons according to both the time delay between their arrival and the laser pulse and their $x-y$ coordinates.

Note that TCSPC is a statistical method that requires high photon counts to analyze the fluorescence decay: The faster the count rate, the lower the acquisition time. The detector count rate is the limiting step for the acquisition time. Indeed, in the case of two photons emitted in a very short time (high intensity of the laser), only the first one will be processed, which will induce artificially faster fluorescence kinetics (shorter lifetime). The benefit of using a very low excitation intensity level (currently $20 \mathrm{nW}$ at the sample) is to avoid photobleaching and/or photoconversion of the FPs (Tramier et al. 2006). Moreover, because of the high sensitivity, very dim cells can be analyzed, avoiding undesirable overexpression effects. Using a high-numerical-aperture (high-NA) $60 \times$ objective, a typical acquisition of an enhanced green FP (eGFP)-labeled cell takes $\sim 2-3$ min.

\section{Multiphoton Multifocal FLIM: TriM-FLIM}

The objective of multifocal multiphoton FLIM (TriM-FLIM) is to combine pseudowide-field pulsed excitation (here in two-photon excitation) and a fast-gated CCD camera (Fig. 3; for an example of an application of two-photon FLIM see also Imaging Intracellular Signaling Using Two-Photon Fluorescent Lifetime Imaging Microscopy [Yasuda 2012]). The principle is to open the intensifier in front of the camera for short times (during the fluorescence decay) after the laser pulse.

Excitation: The excitation source for the TriM-FLIM is a femtosecond-pulsed mode-locked Ti: sapphire laser (Spectra-Physics, France) that is tunable from 700 to $950 \mathrm{~nm}$. The laser is directed into the TriMScope (LaVision Biotec $\mathrm{GmbH}$ ) box, which contains a 50/50 beam splitter and mirrors to divide the incoming laser into from 2 to 64 beams. The set of beams passes through a $2000 \mathrm{~Hz}$ scanner before illuminating the back aperture of a $60 \times$ NA 1.2 infrared water-immersion objective (Olympus). A line of foci is then created at the focal plane, which can be scanned across the sample, producing pseudowide-field illumination.

Detection: A filter wheel of different spectral filters is used to select the fluorescence imaged onto a fast-gated light intensifier connected to a CCD camera (PicoStar, LaVision Biotec GmbH). The gate of the intensifier (adjusted to 1 or $2 \mathrm{nsec}$, depending on the experiment) is triggered by an electronic signal coming from the laser and a programmable delay box, which together are used to acquire a stack of time-correlated images. The number of gates and their time width determine the time resolution of the fluorescence kinetics. The acquisition time for each gate determines the signal-to-noise ratio. The time-correlated gated images are acquired sequentially. Thus, use caution to avoid photobleaching during acquisition, which can artificially shorten the fluorescence decay. 


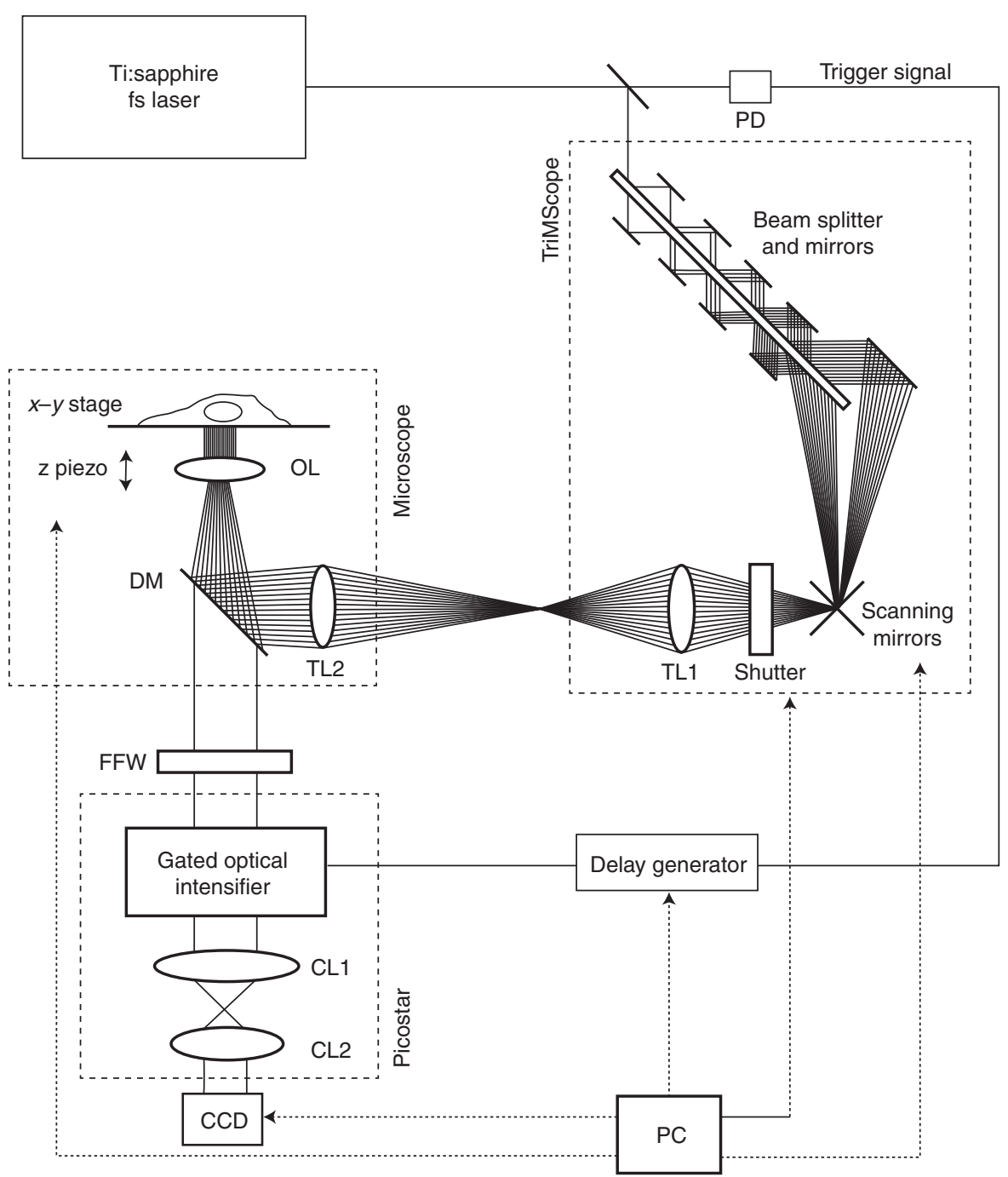

FIGURE 3. Time-domain TriM-FLIM setup for fast acquisitions. PD, photodiode; OL, objective lens; DM, dichroic mirror; TL1, transfer lens 1; TL2, transfer lens 2; FFW, fast filter wheel; CL1, camera lens 1; CL2, camera lens 2; CCD, charge-coupled device; PC, personal computer.

\section{ANALYSIS OF THE FLUORESCENCE DECAYS OF THE DONOR}

The biological information that can be obtained from the fluorescence decay depends on the analysis that is performed. Mainly, the fluorescence decay can be fitted with different models (one or two discrete exponentials or more complex models) or the mean fluorescence lifetime $\tau$ can be obtained without fitting procedures to obtain raw FLIM data. If, in either case, FRET is detected, the biological information will be different in terms of quantification and spatial and temporal resolutions. To obtain quantitative measurements about the fraction of donor molecules interacting with the acceptor, it is necessary to perform a fit of the donor fluorescence decay obtained with a high signal-to-noise ratio, and hence a high photon count, using a two-exponential model. This fitting process requires an acquisition method, which is time consuming (TCSPC method) and impedes the measurement of transient interactions at high spatial resolution. A compromise can be found by using a fast-gated $\mathrm{CCD}$, exploiting the mean fluorescence lifetime of the donor by calculating the minimal fraction of interacting donor $m f_{\mathrm{D}}$ pixel by pixel. Interestingly, the $m f_{\mathrm{D}}$ spatiotemporal variations within a single cell are similar to those of $f_{\mathrm{D}}$ obtained from the fit of the fluorescence decay using a double-exponential model (Padilla-Parra et al. 2008). 
S. Padilla-Parra et al.

Fitting Data from a TCSPC-FLIM System

The fluorescence decay obtained by using the TCSPC method shows a high photon count (binning the pixels over large area) with picosecond time resolution. The least-squares method is a valid way to find mechanistic parameters of the system, such as the fluorescence lifetimes and amplitudes, by using Globals (University of California at Irvine) with a Levenberg-Marquardt algorithm (LMA). Nonlinear LMA for parameter estimation should take into consideration the following hypotheses: (i) Data uncertainties should be related to the fluorescence intensity (photon counts); (ii) fluorescence intensity uncertainties should be Gaussian distributed; (iii) there should not be systematic uncertainties related to all data; (iv) the model used (single exponential [in the case of eGFP alone expressed in live cells]; double exponential, stretched exponential, or Gaussian exponential in all cases) should be a good description of the experimental data; and (v) enough photon counts should be acquired to obtain a good fit.

Among the different models that can be applied to describe the fluorescence intensity decay profile of a related fluorophore, we use a two-species model in which two populations are taken into consideration (an interacting fraction corresponding to a population that relaxes through FRET $\left(f_{\mathrm{D}}\right)$ and a noninteracting fraction in which the donor lifetime remains undisturbed $\left.\left[1-f_{\mathrm{D}}\right]\right)$. The donor lifetime obtained out of the single-exponential fit from cells expressing the donor alone is assigned and is fixed into the noninteracting fraction of the double-exponential model in the corresponding cotransfected cell in which a given protein interaction is under study (Emiliani et al. 2003),

$$
i(t)=\left(1-f_{\mathrm{D}}\right) e^{-\mathrm{t} / \tau_{\mathrm{D}}}+f_{\mathrm{D}} \mathrm{e}^{-\mathrm{t} / \tau_{\mathrm{F}}},
$$

where $f_{\mathrm{D}}$ stands for the fraction of the interacting donor, $\tau_{\mathrm{D}}$ is the fixed donor lifetime, and $\tau_{\mathrm{F}}$ is the discrete FRET lifetime. In this type of analysis, the total intensity $\left(I_{0}\right)$ is normalized to 1 ; and, therefore, both preexponential factors, and particularly $f_{\mathrm{D}}$, vary from 0 to 1 . As an example, Figure 4 depicts the fluorescence decay of eGFP fused to the double bromodomain ([BD]-eGFP) of the transcription factor TAFIID 250, together with the corresponding fits (black line) expressed in a live HeLa cell (green line) in the absence (Fig. 4A) and the presence (Fig. 4B) of mCherry fused to histone H4 (mCherry-H4).

A

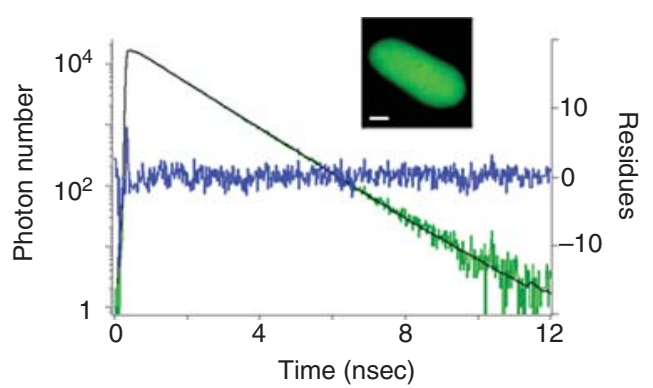

B

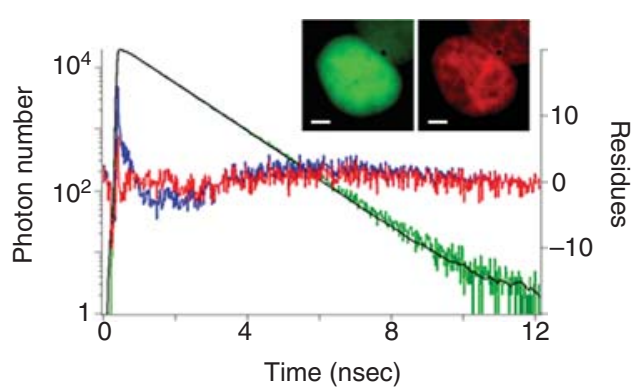

FIGURE 4. eGFP-BD interaction with acetylated mCherry-H4 in the nucleus of HEK293 live cells using the TCSPC method. (A) Steady-state intensity image of eGFP-BD expressed alone in HEK293 cells (green). The corresponding eGFP-BD fluorescence decay (green curve) extracted from the whole nucleus is fitted by a single exponential (black line, $\tau_{\mathrm{D}}=2.59 \mathrm{nsec}$ ), and the residues are presented (blue curve). (B) Steady-state intensity images of eGFPBD (green) and mCherry-H4 (red) coexpressed in HEK293 cells. The corresponding eGFP-BD fluorescence decay (green curve) extracted from the whole nucleus is fitted by a single model $(\tau=2.46 \mathrm{nsec})$ and by a biexponential model $\left(\tau_{\mathrm{D}}=2.59 \mathrm{nsec}\right.$ fixed and $\tau_{\mathrm{F}}=0.65 \mathrm{nsec}$ ) and residues are presented (blue curve and red curve, respectively). Note that the fluorescence decay is better fitted with a biexponential model as shown by residues. Scale bar, $2 \mu \mathrm{m}$. 
Data Treatment Coming from the TriM-FLIM System

With the TriM-FLIM system, the mean fluorescence lifetime is determined for each pixel of the image.

Considering a fluorescence decay $i(t)$, the mean fluorescence $(\tau)$ is defined as

$$
<\tau>=\int t \cdot i(t) \cdot d t / \int i(t) \cdot d t,
$$

in which $t$ is the time. To analyze data coming from a discrete sampling, the mean lifetime is directly calculated by applying Equation 13. For a time-gated stack of images, we have

$$
<\tau>=\Sigma \Delta t_{i} \cdot I_{\mathrm{i}} / \Sigma I_{i}
$$

where $\Delta t_{i}$ corresponds to the time delay after the laser pulse of the $i$ th image acquired and $I_{i}$ corresponds to the pixel intensity map in the $i$ th image (Fig. 5). The map of the mean fluorescence lifetime can be calculated and displayed.

If enough time-gated images are acquired (up to 11), the fluorescence decay can be fitted with an LMA, applying, for example, a discrete two-exponential model as in the case of the TCSPC method, and $\tau_{\mathrm{D}}$ and $\tau_{\mathrm{F}}$ will be determined together with $f_{\mathrm{D}}$.

The fraction of donor that is involved in FRET can be calculated directly from the mean lifetime of the donor $\tau_{\mathrm{D}}$ and $\tau_{\mathrm{F}}$ :

$$
\tau=\left[\left(1-f_{\mathrm{D}}\right) \cdot\left(\tau_{\mathrm{D}}^{2}+f_{D} \cdot \tau_{\mathrm{F}}^{2}\right] /\left[\left(1-f_{\mathrm{D}}\right) \cdot \tau_{\mathrm{D}}+f_{\mathrm{D}} \cdot \tau_{\mathrm{F}}\right] .\right.
$$

Isolating $f_{\mathrm{D}}$ and normalizing the last expression by dividing by $\tau_{\mathrm{D}}$, we find an expression that accounts for the fraction of interacting donor:

$$
f_{\mathrm{D}}=\left[1-\left(<\tau>/ \tau_{\mathrm{D}}\right)\right] /\left[1-\left(\tau / \tau_{\mathrm{D}}\right)-\left(\tau_{\mathrm{F}} / \tau_{\mathrm{D}}\right)^{2}+\left(\tau / \tau_{\mathrm{D}}\right) \cdot\left(\tau_{\mathrm{F}} / \tau_{\mathrm{D}}\right)\right]
$$

Maps of $\tau$ and of $f_{\mathrm{D}}$ (assuming the same $\tau_{\mathrm{F}}$ value for each pixel) are obtained as shown in Figure 6, in which the interaction between BD-eGFP and mCherry-H4 is visualized in the nucleus of living cells by the decrease of the fluorescence lifetime of eGFP-BD compared with that of eGFP-BD in the absence of mCherry-H4 (monotransfected cells, the mean lifetime averaged throughout the nucleus decreased from $2.41 \mathrm{nsec}$ for the control to $2.34 \mathrm{nsec}$ for the cotransfection). The fraction of eGFP-BD interacting with mCherry-H4 can be determined and mapped within the nucleus.

\section{The Minimal Fraction of Interacting Donor}

The minimal fraction of interacting donor, $m f_{\mathrm{D}}$ (Padilla-Parra et al. 2008), is calculated directly from the values of the mean fluorescence lifetime of the donor in the absence and in the presence of the acceptor.

Mathematically, $f_{\mathrm{D}}$ depends on two variables $\left(t_{\mathrm{F}}\right.$ and $\left.t\right)$ and can be minimized following $t_{\mathrm{F}}$ :

$$
m f_{\mathrm{D}}=\left[1-\left(\tau / \tau_{\mathrm{D}}\right)\right] /\left[\left(\tau / 2 \cdot \tau_{\mathrm{D}}\right)-1\right]^{2} .
$$

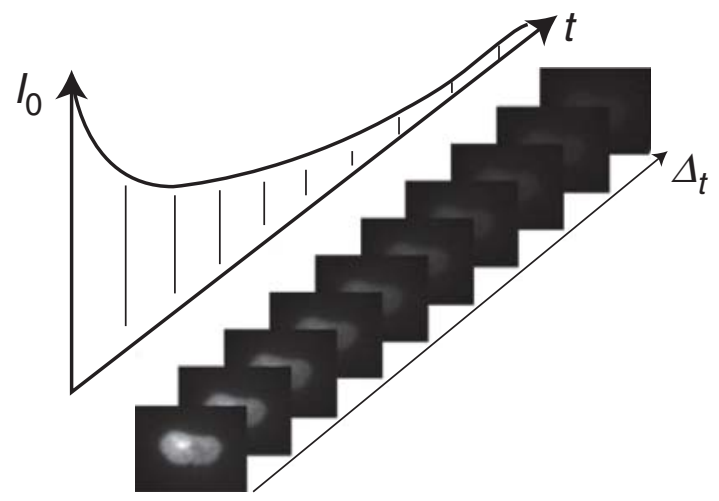

FIGURE 5. Cartoon representing a sequential acquisition of images from the time-domain TriM-FLIM setup. 
S. Padilla-Parra et al.
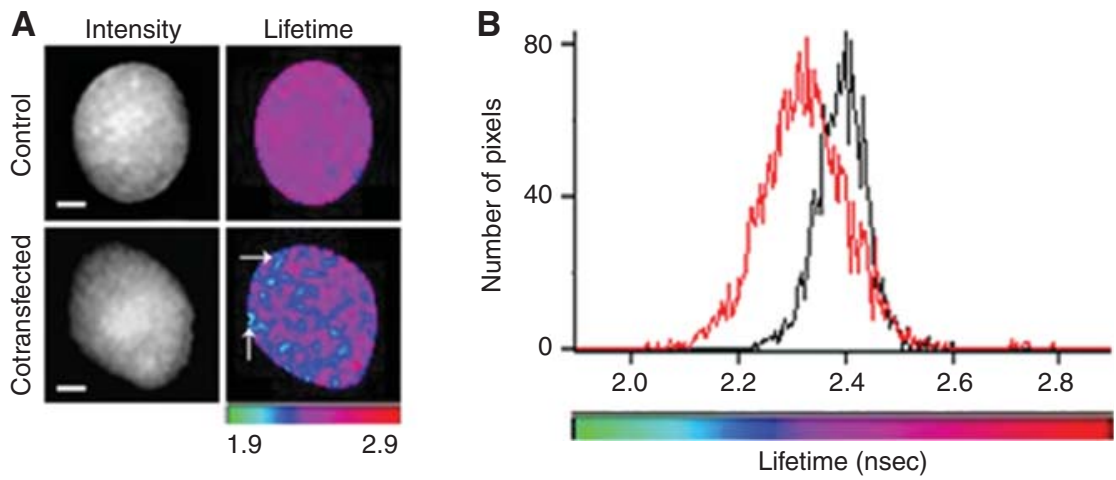

FIGURE 6. Intensity and FLIM images of eGFP-BD expressed alone as the control or with mCherry-H4 as the cotransfection in HEK293 live cells using a TriM-FLIM system and 11 time-gated images. (A) Intensity images were obtained by summing the time-gated stack. FLIM images were obtained by using Equation 14 in a pixel-by-pixel manner. White arrows show two chromatin domains in which eGFP-BD mean lifetime decreases significantly. $(B)$ Histograms of the mean fluorescence lifetime for the control (black line) and the cotransfected cell (red line). The mean lifetime averaged throughout the nucleus decreased from $2.41 \mathrm{nsec}$ for the control to $2.34 \mathrm{nsec}$ for the cotransfection. Scale bar, $2 \mu \mathrm{m}$.

$m f_{\mathrm{D}}$ provides instantaneous knowledge about the minimal extent of the interaction under study. This is particularly relevant in biology because, without knowing the intrinsic transfer efficiency (because $m f_{\mathrm{D}}$ does not require previous knowledge of $\tau_{\mathrm{F}}$ ), quantitative data related to the relative concentration are immediately at hand.

The impact of fast acquisitions of $f_{\mathrm{D}}$ and $m f_{\mathrm{D}}$ with the same biological example (eGFP-BD and mCherry-H4) was investigated by using the TriM-FLIM system (Fig. 7). On the same cells, two windowing schemes were chosen, one using only five time-gated images ( $2 \mathrm{nsec}$ gate width) for very fast acquisition times $(\sim 3 \mathrm{sec})$ and the other using 11 time-gated images (1 nsec gate width) for longer acquisition times $(\sim 30 \mathrm{sec})$.

$f_{\mathrm{D}}$ was calculated from the value of $\tau_{\mathrm{F}}$ determined from the biexponential fit of the fluorescence decay of the donor in the presence of the acceptor from the stack of 11 time-gated images $\left(\tau_{\mathrm{F}}=0.65 \pm\right.$ $0.59 \mathrm{nsec}, n=33)$. The donor lifetime was fixed to $\tau_{\mathrm{D}}=2.40 \mathrm{nsec}$, coming from the monoexponential fit of the donor alone. A $3 \mathrm{D}$ representation of both $f_{\mathrm{D}}$ and $m f_{\mathrm{D}}$ images using a threshold limit given by the control is included to reveal the differences between control and FRET images (Fig. 7). Both $f_{\mathrm{D}}$ and $m f_{\mathrm{D}}$ images are very similar and present the same pattern when FRET occurs with a very close mean value of $f_{\mathrm{D}}(0.13)$ and $m f_{\mathrm{D}}(0.11)$. The two histograms are also superimposed in Figure 7 (right panel). The underestimation of the amount of donor that undergoes FRET when taking $m f_{\mathrm{D}}$ relative to $f_{\mathrm{D}}$ is only $15 \%$ in this example.

Number of Interacting Particles and Relative Concentration

When we are interested in following the spatiotemporal changes of a highly localized protein, it is convenient to use a different approach related to the relative concentration: the number of interacting particles (NP). Assuming a two-species model and single-exponential behavior of the donor, fluorescence intensity as a function of time can be defined as

$$
i(t)=I_{0} f_{\mathrm{D}} \cdot e^{-t / \tau_{\mathrm{F}}}+I_{0}\left(1-f_{\mathrm{D}}\right) \cdot e^{-\mathrm{t} / \tau_{\mathrm{D}}},
$$

where $I_{0}$ is the fluorescence intensity at time 0 . All of the other parameters have already been defined. Note that here, $i(t)$ is not normalized to 1 , and its amplitude corresponds to the relative concentration of fluorophores:

$$
i(t)=k_{\mathrm{r}}\left([D A] \cdot e^{-t / \tau_{\mathrm{F}}}+[D] \cdot e^{-t / \tau_{\mathrm{D}}}\right),
$$



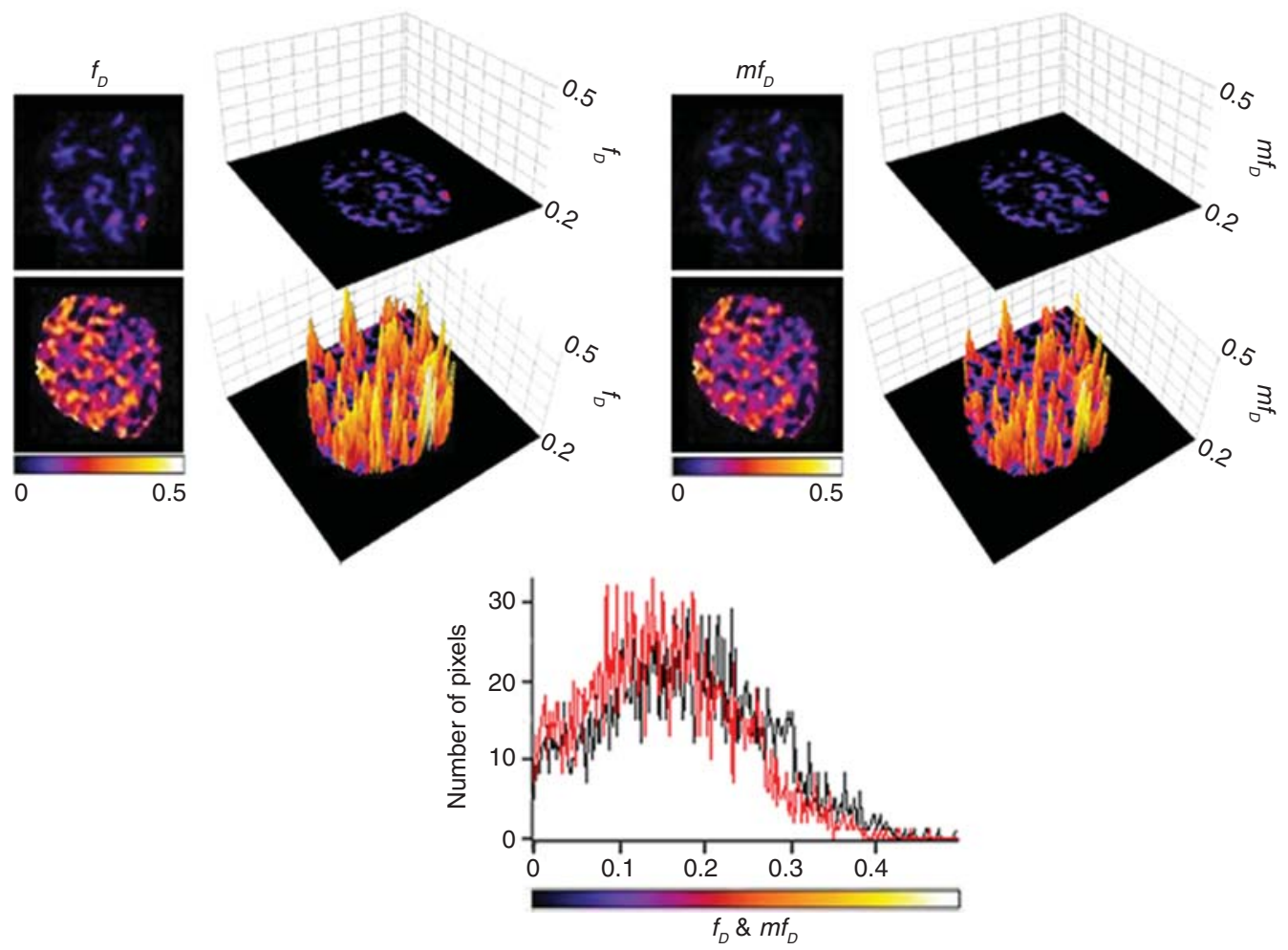

FIGURE 7. (Top) $f_{\mathrm{D}}$ and $m f_{\mathrm{D}}$ images obtained by using Equation 16 (with $\tau_{\mathrm{D}}=2.41 \mathrm{nsec}$ and $\tau_{\mathrm{F}}=0.65 \mathrm{nsec}$ ) and Equation 17 (with $\tau_{\mathrm{D}}=2.41 \mathrm{nsec}$ ), respectively. Three-dimensional representation of the corresponding $f_{\mathrm{D}}$ and $m f_{\mathrm{D}}$ images using a threshold limit given by the control (0.2) are also presented. (Bottom) The comparison between the histogram coming from $f_{\mathrm{D}}$ and $m f_{\mathrm{D}}$ clearly show that, for this example, both values behave similarly for all pixels of the image. Note that this is the same cell showed in Figure 6 and the dimensions of the micrograph are $12 \times 12 \mu \mathrm{m}$.

where $k_{\mathrm{r}}$ is the rate constant of the radiative process, $[\mathrm{DA}]$ is the concentration of the interacting complex, and $[\mathrm{D}]$ is the concentration of the noninteracting donor population. In Equation 19, [DA] is proportional to $\left(I_{0} \times f_{\mathrm{D}}\right)$. Note that we can approximate $f_{\mathrm{D}}$ by using the $m f_{\mathrm{D}}$ image and $I_{0}$ by using the corresponding first gated image. We present a biological example corresponding to the activation of Rac followed by FRET occurring between eGFP-Rac and PBD-mCherry (which is the binding domain of an effector of Rac fused to mCherry) and applying the number of particles in Figure 8 (see online Movie 1 at cshprotocols.cshlp.org, which shows a time lapse of the spatiotemporal activation of Rac [relative $\mathrm{NP}=m f_{\mathrm{D}} \times I_{0}$ ], and online Movie 2 at cshprotocols.cshlp.org, which shows a time lapse of the corresponding eGFP-Rac fluorescence intensity).

\section{PRACTICAL CONSIDERATIONS}

Limitations and Use of $m f_{\mathrm{D}}$

The use of $m f_{\mathrm{D}}$ described here is applicable if we consider a two-component system, the unbound donor and donor involved in FRET with the acceptor with a narrow distribution of $E$. Although one of the constraints mentioned above deals with the single-exponential behavior of the donor (e.g., eGFP), the $m f_{\mathrm{D}}$ concept can be extended to multiexponential donors, such as cyan FP (CFP) (Padilla-Parra et al. 2008).

\section{Choice of the Best FRET Couple}

The most important characteristics of good FRET-FLIM donors are that (i) their fluorescence decay profile must be fit best with a single-exponential model, (ii) their fluorescence intensity as a function 
S. Padilla-Parra et al.

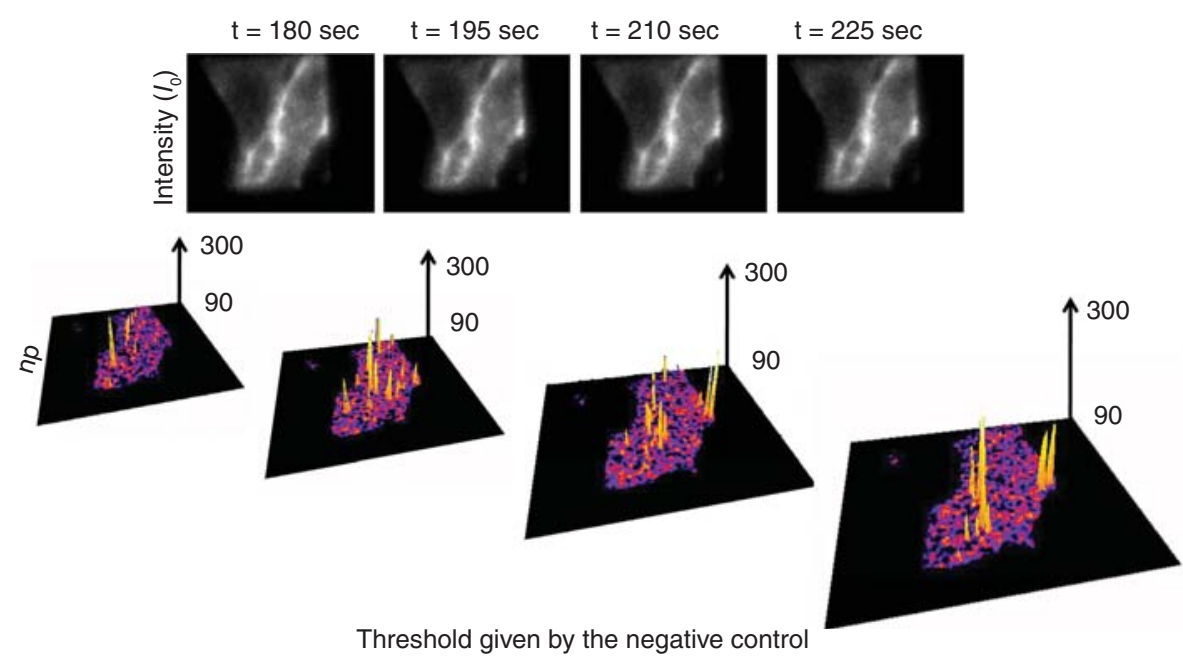

FIGURE 8. Time lapse of Rac activation followed by fast FLIM. A cotransfected cell with eGFP-Rac and Pbd-mCherry shows a transient activation localized preferentially in subcellular domains at the periphery of the cell. (Top) The first time-gated intensity image of a sequential acquisition of five time-gated images ( 2 nsec each) is presented (see online Movie 2 at cshprotocols.cshlp.org). (Bottom) The corresponding NP images are shown with a threshold given by the negative control (eGFP-Rac expressed alone coming from a cell with the same signal-to-noise ratio) (see online Movie 1 at cshprotocols.cshlp.org). The NP is determined by the product of the first gated image and the $m f_{\mathrm{D}}$ value, as described in the text. The Rac activation (bottom) is independent of the eGFP-Rac fluorescence intensity (top). The dimensions of the micrograph are $16 \times 15 \mu \mathrm{m}$.

of time should remain constant under different illumination conditions (i.e., they have good photostability), and (iii) they should not be susceptible to undergoing photoconversion.

mTFP1 is a monomeric cyan protein whose fluorescence decay is best described with a singleexponential model (Ai et al. 2006; Padilla-Parra et al. 2009) as is eGFP (Tramier et al. 2006). Different FRET pairs were tested by linking two different FPs with a polypeptide chain (tandem). By using TCSPC-FLIM, we compared different tandems (Table 1). The relatively low $f_{\mathrm{D}}$ percentages could come from a possible spectroscopic heterogeneity of the acceptor population, which is partially caused by different maturation rates for the donor and the acceptor (Padilla-Parra et al. 2009).

The usefulness of mTFP1-enhanced yellow FP (eYFP) as a FRET couple is supported by the twofold increase of the minimal fraction of interacting histone $\mathrm{H} 4$ with the double BD of TAFII 250, when using mTFP1-H4/eYFP-BD instead of eGFP-H4/mCherry-BD using the TriM-FLIM (Fig. 9). Note that mTFP1 is fairly resistant to photobleaching. However, attention should be paid to the effect of photobleaching when performing time-lapse TriM-FLIM acquisition with fusion proteins using mTFP1 in biological systems in which the proteins are highly immobile, as is the case for histone $\mathrm{H} 4$ incorporated into chromatin.

\section{Effect of Photobleaching on FRET Determination: False FRET Signals}

The existence of photobleaching and/or photoconversion before or during the acquisition can induce false-positive FRET determination. In steady-state measurements, especially with the CFP/YFP

TABLE 1. Intrinsic FRET efficiency $(E)$ and the fraction of interacting donor $\left(f_{\mathrm{D}}\right)$ for a set of different tandem constructs using a two-species model with a double-exponential model fixing the donor to a previously calculated value $N=5$

\begin{tabular}{lcr}
\hline Tandem & $f_{\mathrm{D}}$ & $E$ \\
\hline mRFP1-eGFP & $0.26 \pm 0.08$ & $0.56 \pm 0.02$ \\
mStrawberry-eGFP & $0.37 \pm 0.07$ & $0.58 \pm 0.02$ \\
mCherry-eGFP & $0.45 \pm 0.02$ & $0.58 \pm 0.03$ \\
mTFP1-mOrange & $0.37 \pm 0.01$ & $0.68 \pm 0.02$ \\
mTFP1-eYFP & $0.71 \pm 0.01$ & $0.61 \pm 0.08$ \\
\hline
\end{tabular}

RFP, red fluorescent protein; eGFP, enhanced green fluorescent protein; eYFP, enhanced yellow fluorescent protein. 
A

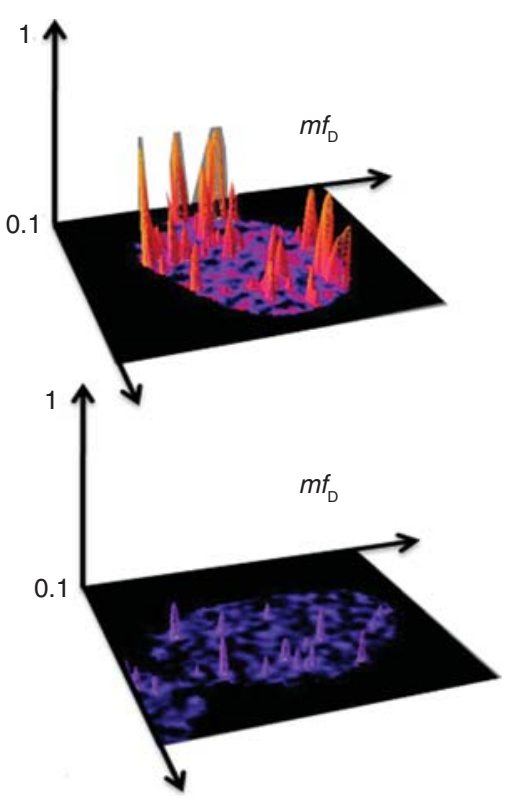

B

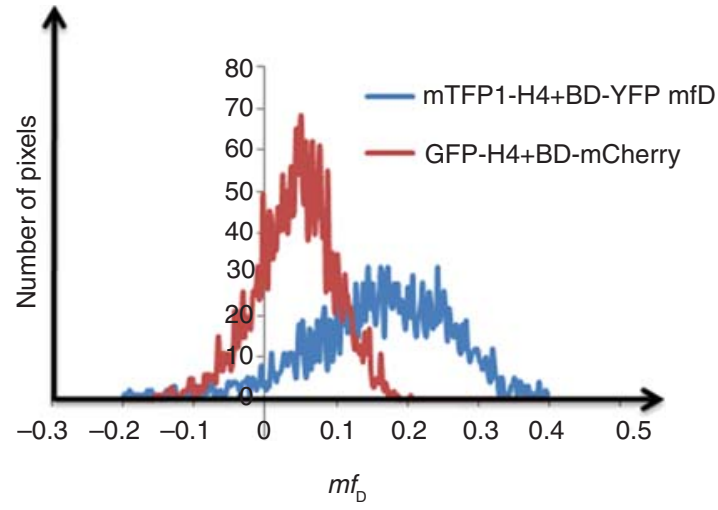

FIGURE 9. $m f_{\mathrm{D}}$ comparison between the FRET couples mTFP1/eYFP and eGFP/mCherry from fast-FLIM acquisitions. (A) Three-dimensional maps of $m f_{\mathrm{D}}$ for mTFP1-H4 coexpressed with eYFP-BD (upper panel) and eGFP-H4 coexpressed with $\mathrm{mCherry-BD}$ (lower panel). $(B)$ The overlapping $m f_{\mathrm{D}}$ distribution for both examples is shown as well as the higher average $m f_{\mathrm{D}}$ for the mTFP-H4+ eYFP-BD couple. The dimensions of both micrographs are $14 \times 14 \mu \mathrm{m}$.

(yellow fluorescent protein) couple, donor photobleaching (CFP is more sensitive to excitation light than YFP) or photoconversion of YFP into a CFP-like species (with the acceptor photobleaching method) results in a ratio of CFP and YFP intensity similar to what can be obtained in a FRET situation (Valentin et al. 2006). The excitation light intensity is much lower with the TCSPC method; and thus this method is less prone to photobleaching. However, photobleaching can occur before the acquisition when observing and choosing the cells. Importantly, when using fast acquisitions (e.g., TriM-FLIM or any other sequential method for the fluorescence lifetime measurement; Grant et al. 2007), the occurrence of photobleaching on single-exponential fluorophores during the acquisition of FLIM has a drastic effect on the mean fluorescence lifetime, which is not the case with TCSPC (Tramier et al. 2006; Padilla-Parra et al. 2009). When acquiring images very quickly (e.g., by taking five time-gated images), if photobleaching occurs, the intensity of the fifth image can be too weak to have a significant value. This phenomenon shortens the mean lifetime determination as in the FRET situation, causing a bias in the interpretation of results.

Reasons for the Absence of a FRET Signal

The occurrence of FRET with a particular FP couple relies on the close proximity of the donor and the acceptor $(R<80 \AA)$ and on the correct orientation of their dipoles (they cannot be perpendicular to each other). In addition to these basic requirements, FRET cannot be detected in certain situations even if the corresponding endogenous proteins interact to some extent. The classic reason for the absence of FRET is the localization of the fluorescent tag to a position of the protein sequence that impedes the interaction with its partners. More often encountered is the situation in which the amount of interacting donor per pixel is very weak in front of the noninteracting donor; therefore, it is difficult to get a significant FRET signal. We observe this situation, for example, in polymeric protein structures, such as actin filaments or microtubules. Hetero-FRET detection arises from the close proximity of a donor-tagged and an acceptor-tagged monomer within the polymer. The occurrence of this situation is statistically weak, and no FRET is detected. A related situation, in which it is difficult to unveil FRET, occurs when the interactions between the proteins of interest are transient 
and subject to spatiotemporal fluctuations, as we saw in the examples above. In this situation, fast time-lapse FLIM has to be performed to avoid the average of the FRET signals in space and time. The existence of a big proportion of immature red acceptor (up to 60\%) artificially decreases the amount of bound donor. All these considerations show the difficulties (and likely the impossibility) of obtaining true quantitative measurements for the amount of donor protein that is interacting when we carry out live cell studies. This state of affairs reinforces the use of the concept of the minimal fraction of interacting donor protein $m f_{\mathrm{D}}$. When using bioprobes based on intramolecular FRET (such as Raichu or other tandem probes used to monitor changes in biochemical activity), the steady-state intensity ratio measurements can be competitive in front of the fast FRET-FLIM method. However, for protein-protein interaction measurements (intermolecular FRET with no control on the amount of each partner within live cells), determining $m f_{\mathrm{D}}$ by using fast FRET-FLIM (time or frequency domain) is the best method because it is independent of the local concentration of protein.

\section{CONCLUSIONS}

The classical analysis FLIM technique in which the fluorescent decay is fitted to each pixel of a related image remains challenging when it is used to monitor protein-protein interactions in live cells. Compromises must be made between the precision and the time invested in the measurement. We have shown a simple way to analyze data derived from setups based on TCSPC detection, and we have also provided an alternative way to quantify transient protein interactions with faster systems based on sequential acquisitions. In that sense, the $m f_{\mathrm{D}}$ approach is an original method to quantify protein interactions for very fast acquisitions in which low photon counts and time points are required. This method is capable of providing information related to transient interactions in live cells.

\section{ACKNOWLEDGMENTS}

We thank Jean Claude Mevel, Dr. Marie Jo Masse, Dr. Allison Marty, and Dr. Guy Van Tran Nhieu for technical assistance with plasmid constructs. Work described in this introduction was performed at the Imaging Center (ImagoSeine) of the Institut Jacques Monod and was supported by the Fondation pour la Recherche Medicale, the Region Ile de France (Soutien aux Equipes Scientifiques pour l'Acquisition de Moyens Expérimentaux), the Centre National de la Recherche Scientifique (Action Concertee Incitative Biologie Cellulaire, Moleculaire et Structurale), the Association pour la Recherche sur le Cancer, and the Association Nationale pour le Recherche. S.P.-P. is a recipient of a European Union predoctoral fellowship (Marie-Curie Grant No. MRTN-CT 2005-019481).

\section{REFERENCES}

Ai HW, Henderson JN, Remington SJ, Campbell RE. 2006. Directed evolution of a monomeric, bright and photostable version of Clavularia cyan fluorescent protein: Structural characterization and applications in fluorescence imaging. Biochem J 400: 531-540.

Berney C, Danuser G. 2003. FRET or no FRET: A quantitative comparison. Biophys J 84: 3992-4010.

Cheung HC. 1991. Resonance energy transfer. In Topics of fluorescence microscopy, Volume 2, Principles (ed. Lakowicz JL), Chapter 3, pp. 123-176. Plenum, New York.

Dale RE, Eisinger J, Blumberg WE. 1979. The orientational freedom of molecular probes: The orientation factor in intramolecular energy transfer. Biophys J 26: 161-194. Errata: 30: 365.

Elangovan M, Wallrabe H, Che Y, Day RN, Barroso M, Periasamy A. 2003. Characterization of one- and two-photon excitation fluorescence resonance energy transfer microscopy. Methods 29: 58-73.

Emiliani V, Sanvitto D, Tramier M, Piolot T, Petrasek Z, Kemnitz K, Durieux C, Coppey-Moisan M. 2003. Low intensity two-dimensional imaging of fluorescence lifetimes in living cells. Appl Phys Lett 83: $2471-2473$.

Förster T. 1948. Intermolecular energy migration and fluorescence. Ann Phys 6: 55-75.

Gordon GW, Berry G, Liang XH, Levine B, Herman B. 1998. Quantitative fluorescence resonance energy transfer measurements using fluorescence microscopy. Biophys J 74: 2702-2713.

Grant DM, McGinty J, McGhee EJ, Bunney TD, Owen DM, Talbot CB, Zhang W, Kumar S, Munro I, Lanigan PMP, et al. 2007. High speed optically sectioned fluorescence lifetime imaging permits study of live cell signaling events. Opt Express 15: 15656-15673.

Hoppe A, Christensen K, Swanson JA. 2002. Fluorescence resonance energy transfer-based stoichiometry in living cells. Biophys J 83: 3652-3664.

Lakowicz JR. 2006. Principles of fluorescence spectroscopy, 3rd ed. Springer, New York.

Neher RA, Neher E. 2004. Applying spectral fingerprinting to the analysis of FRET images. Microsc Res Tech 64: 185-195. 
Padilla-Parra S, Audugé N, Coppey-Moisan M, Tramier M. 2008. Quantitative FRET analysis by fast acquisition time domain FLIM at high spatial resolution in living cells. Biophys J 95: 2976-2988.

Padilla-Parra S, Audugé N, Coppey-Moisan M, Tramier M. 2009. Quantitative comparison of different fluorescent protein couples for fast FRET-FLIM acquisition. Biophys J 97: 2368-2376.

Peter M, Ameer-Beg SM, Hughes MKY, Keppler MD, Prag S, Marsh M, Vojnovic B, Ng T. 2005. Multiphoton-FLIM quantification of the eGFP-mRFP1 FRET pair for localization of membrane receptorkinase interactions. Biophys J 88: 1224-1237.

Shaner NC, Steinbach PA, Tsien RY. 2005. A guide to choosing fluorescent proteins. Nat Methods 2: 905-909.

Stockholm D, Bartoli M, Sillon G, Bourg N, Davoust J, Richard I. 2004. Imaging calpain protease activity by multiphoton FRET in living mice. J Mol Biol 346: 215-222.

Tramier M, Zahid M, Mevel JC, Masse MJ, Coppey-Moisan M. 2006. Sensitivity of CFP/YFP and GFP/mCherry pairs to donor photobleaching on FRET determination by fluorescence lifetime imaging microscopy in living cells. Microsc Res Tech 11: 933-942.

Tsien RY. 1998. The green fluorescence protein. Аnnu Rev Biochem 67: $509-524$.
Tuszynski J, Portet S, Dixon J. 2006. Nonlinear assembly kinetics and mechanical properties of biopolymers. Nonlinear Anal 63: 915-925.

Valentin G, Verheggen C, Piolot T, Neel H, Coppey-Moisan M, Bertrand E. 2006. Photoconversion of YFP into a CFP-like species during acceptor photobleaching FRET experiments. Nat Methods 3: 491-492.

Valeur B. 2002. Molecular fluorescence: Principles and applications Wiley$\mathrm{VCH}$, Weinheim, Germany.

van Rheenen J, Langeslag M, Jalink K. 2004. Correcting confocal acquisition to optimize imaging of fluorescence resonance energy transfer by sensitized emission. Biophys J 86: 2517-2529.

Verveer PJ, Wouters FS, Reynolds AR, Bastiaens PIH. 2000. Quantitative imaging of lateral ErbB1 receptor signal propagation in the plasma membrane. Science 290: 1567-1570.

Wlodarczyk J, Woehler A, Kobe F, Ponimaskin E, Zeug A, Neher E. 2008. Analysis of FRET signals in the presence of free donors and acceptors. Biophys J 94: 986-1000.

Yasuda R. 2012. Imaging intracellular signaling using two-photon fluorescent lifetime imaging microscopy. Cold Spring Harb Protoc doi: 10.1101/ pdb.top072090.

Zal T, Gascoigne NRJ. 2004. Photobleaching-corrected FRET efficiency imaging of live cells. Biophys J 86: 3923-3939. 


\section{Time-Domain Fluorescence Lifetime Imaging Microscopy: A Quantitative Method to Follow Transient Protein-Protein Interactions in Living Cells}

Sergi Padilla-Parra, Nicolas Audugé, Marc Tramier and Maïté Coppey-Moisan

Cold Spring Harb Protoc; doi: 10.1101/pdb.top086249

\begin{tabular}{|c|c|}
\hline $\begin{array}{l}\text { Email Alerting } \\
\text { Service }\end{array}$ & Receive free email alerts when new articles cite this article - click here. \\
\hline $\begin{array}{l}\text { Subject } \\
\text { Categories }\end{array}$ & $\begin{array}{l}\text { Browse articles on similar topics from Cold Spring Harbor Protocols. } \\
\text { Cell Biology, general (1382 articles) } \\
\text { Cell Imaging (525 articles) } \\
\text { Fluorescence (517 articles) } \\
\text { Fluorescence, general (341 articles) } \\
\text { Imaging/Microscopy, general (579 articles) } \\
\text { In Vivo Imaging ( } 334 \text { articles) } \\
\text { Live Cell Imaging (274 articles) } \\
\text { Protein Expression and Interactions (93 articles) } \\
\text { Visualization (524 articles) } \\
\text { Visualization of Proteins (107 articles) }\end{array}$ \\
\hline
\end{tabular}

